Original paper

\title{
Galectin-3 is not useful for hepatocellular carcinoma surveillance in cirrhotic patients but it may be a marker of cirrhosis development
}

\author{
Eman Saad Nassar ${ }^{1}$, Yomna Abdelrazek Elkalbashawy ${ }^{1}$, Ahmed Kamal ${ }^{2}$, Nermine Hossam Eldin Zakaria ${ }^{1}$ \\ 'Clinical and Chemical Pathology, Alexandria University, Egypt \\ ${ }^{2}$ Hepatology Unit, Internal Medicine Department, Faculty of Medicine, Alexandria University, Egypt
}

\begin{abstract}
Aim of the study: Ultrasound surveillance for hepatocellular carcinoma $(\mathrm{HCC})$ among cirrhotic patients is the currently used modality but it is operator dependent. Combining a tumor marker with ultrasound may improve sensitivity for early HCC detection. Our aim was to assess the galectin-3 level among HCC and cirrhotic patients on top of chronic hepatitis $\mathrm{C}$ to evaluate its possible role as a tumor marker for $\mathrm{HCC}$ surveillance among cirrhotic patients.

Material and methods: The study was conducted on 160 subjects. They were grouped as follows: group 1: $40 \mathrm{pa}-$ tients with HCC secondary to liver cirrhosis on top of chronic hepatitis C; group 2: 40 patients with cirrhosis secondary to chronic hepatitis C; group 3: 40 patients with chronic hepatitis $\mathrm{C}$ without advanced fibrosis; group 4: 40 healthy controls. Serum galectin-3 levels were determined in all subjects using ELISA.

Results: Serum galectin-3 level was significantly higher in HCC patients than in those with chronic hepatitis $C$ $(p<0.001)$. Also it was significantly higher among cirrhotic patients than in patients with chronic hepatitis $C$ $(p<0.001)$. But on comparing HCC patients with cirrhotic patients, serum galectin-3 levels were not significantly different $(p=0.926)$.

Conclusions: Galectin-3 levels cannot be used as an additional method for surveillance of HCC among cirrhotic patients.
\end{abstract}

Key words: antiviral agents, liver cirrhosis, neoplasm recurrence.

Address for correspondence:

Dr. Ahmed Kamal, Hepatology Unit, Internal Medicine Department, Faculty of Medicine, Alexandria University, Egypt, e-mail: ahmed.kamal@alexmed.edu.eg

\section{Introduction}

Hepatocellular carcinoma (HCC) is one of the prime causes of cancer-related deaths globally [1] and the most frequent cancer in Egypt, comprising 23.8\% of all malignancy cases, because of high hepatitis $\mathrm{C}$ virus (HCV) prevalence [2].

Liver cirrhosis is the main etiology for HCC [3]. Early detection of HCC is important to achieve better management outcomes [4]. Ultrasound surveillance for HCC among cirrhotic patients is the currently used modality but it is operator-dependent and not without limitations
[5]. Combining a tumor marker with ultrasound aims to improve sensitivity for early HCC detection [5].

Galectin-3 is known to affect HCC cell apoptosis, adhesion, migration, angiogenesis, and inflammatory responses, making it a possible tumor marker in HCC [6]. Also, it was discovered to be associated with worse prognosis in HCC patients $[7,8]$ and it can be a target for new therapies for HCC $[9,10]$.

Our aim was to assess the galectin-3 level among HCC and cirrhotic patients with chronic hepatitis C to identify its possible role as a tumor marker for HCC surveillance among cirrhotic patients. 


\section{Material and methods}

\section{Materials}

Sample size was calculated postulating that the mean level of galectin-3 in the HCC group was $4.28 \mathrm{ng} / \mathrm{ml}(\mathrm{SD}=3.16)$ and $2.67 \mathrm{ng} / \mathrm{ml}(\mathrm{SD}=1.61)$ in the cirrhotic group without HCC [11]. It was estimated that 39 patients needed to be included for cirrhosis and HCC groups at 95\% confidence interval (CI) (twosided) and $80 \%$ power. The galectin-3 level in chronic hepatitis $\mathrm{C}$ without advanced fibrosis was assumed to be significantly lower than that in cirrhotic and HCC patients.

The study was conducted on 160 subjects. They were grouped as follows:

- group 1 (HCC group): 40 patients with HCC secondary to liver cirrhosis on top of chronic hepatitis C;

- group 2 (cirrhosis group): 40 subjects with cirrhosis secondary to chronic hepatitis C. All with acoustic radiation force impulse imaging (ARFI) score $\geq 2.2 \mathrm{~m} / \mathrm{s}$ plus either FIB-4 [12] > 3.25 or FIB-4 $>1.45$ with clinical and imaging evidence of cirrhosis. HCC was excluded using triphasic CT;

- group 3 (chronic hepatitis $\mathrm{C}$ group): 40 subjects with chronic hepatitis $\mathrm{C}$ without advanced fibrosis (with no clinical or sonographic evidence of liver cirrhosis plus FIB- $4<1.45$ and ARFI score $<1.4 \mathrm{~m} / \mathrm{s}$ );

- group 4: 40 healthy controls.

\section{Methods}

Data were collected regarding history, clinical findings, complete blood count (CBC), serum aspartate and alanine aminotransferases (AST and ALT), serum bilirubin, serum albumin, prothrombin activity, international normalized ratio (INR), $\alpha$-fetoprotein (AFP), HCV antibodies, HBsAg and hepatitis B core antibody ( $\mathrm{HBcAb})$ using ELISA, and HCV RNA levels in serum using real-time PCR assay. Liver disease severity was determined depending on the modified Child-Pugh score [13] and Model for End-Stage Liver Disease (MELD) score [13]. Imaging assessment relied on ultrasonography and ARFI score measurement. A triphasic computed tomography (CT) liver scan was carried out within 12 weeks prior to blood samples' withdrawal for all cirrhotic patients. Patients with LR5 [14] lesions were included in group 1 while in group 2 we included only patients without evidence of any focal hepatic lesion.

Serum galectin-3 levels were verified in all subjects using ELISA (Shanghai Coon Koon Biotech., Ltd) complying with the manufacturer's instructions.
The study was performed in alignment with the revised Declaration of Helsinki (2013), and with Good Clinical Practice guidelines. Our study was approved by the Ethical Committee of the Faculty of Medicine, Alexandria University (IRB No. 00012098). Informed consent from all subjects included in the study was obtained.

\section{Statistical analysis}

Data were analyzed using IBM SPSS software package version 20.0. (Armonk, NY: IBM Corp). Significance of the obtained results was judged at the $5 \%$ level. The $\chi^{2}$ test was used for categorical variables, to compare between different groups. The F-test (ANOVA) was used for normally distributed quantitative variables, to compare between more than two groups, and a post hoc test (Tukey) for pairwise comparisons. The Kruskal-Wallis test was used for non-normally distributed quantitative variables, to compare between more than two studied groups, and a post hoc test (Dunn's multiple comparisons test) for pairwise comparisons.

\section{Results}

There was no significant differences in age between HCC and cirrhotic patients ( $p=0.238$ ), or between cirrhotic and chronic viral hepatitis $C$ patients $(p=0.166)$. Age was significantly higher in the HCC group than the chronic viral hepatitis group $(p=0.001)$.

In the HCC group, the mean serum albumin level was $2.44 \pm 0.6 \mathrm{~g} / \mathrm{dl}$, and in the cirrhotic group mean serum albumin was $2.66 \pm 0.73 \mathrm{~g} / \mathrm{dl}$, with no statistically significant difference between the groups $(p=0.464)$. In the chronic hepatitis $\mathrm{C}$ group the mean serum albumin level was $3.63 \pm 0.73 \mathrm{~g} / \mathrm{dl}$ with a statistically significant difference with the cirrhotic group $(p<0.001)$.

In the HCC group, the median total bilirubin level was $1.80 \mathrm{mg} / \mathrm{dl}(1.2-5.25)$. In the cirrhotic group, the median was $1.1 \mathrm{mg} / \mathrm{dl}(0.8-2.25)$, while in the chronic hepatitis $\mathrm{C}$ group the median was $0.60 \mathrm{mg} / \mathrm{dl}(0.4-0.8)$. The differences were statistically significant.

In the HCC group, the mean platelet count was $96.5 \pm 39.74 \times 10^{3}$ cells $/ \mathrm{mm}^{3}$, while in the cirrhotic group, it was $102.85 \pm 63.08 \times 10^{3}$ cells $/ \mathrm{mm}^{3}(p=0.975)$. In the chronic hepatitis $\mathrm{C}$ group the mean was 280.22 $\pm 83.21 \times 10^{3}$ cells $/ \mathrm{mm}^{3}$, which was significantly different from the cirrhotic group $(p<0.001)$.

As regard AFP, the median in the HCC group was $37.6 \mathrm{ng} / \mathrm{ml}$ (6.75-84), which was significantly higher from that of the cirrhotic group, which was $4.05 \mathrm{ng} / \mathrm{ml}$ (2.3-6.2) $(p<0.001)$, while in the chronic hepatitis $\mathrm{C}$ group it was $3.70 \mathrm{ng} / \mathrm{ml}(1.1-4.9)$ and in the healthy group it was $1.90 \mathrm{ng} / \mathrm{ml}$ (1-3.05). The difference be- 


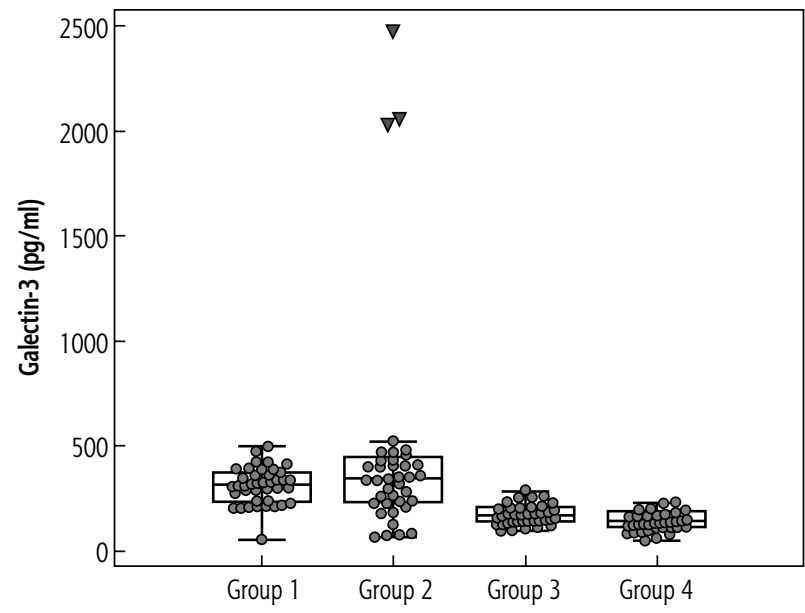

Fig. 1. Comparison between studied groups according to galectin-3 levels

tween cirrhotic and chronic hepatitis $C$ groups was not statistically significant $(p=0.97)$ while AFP was significantly lower in healthy subjects than in chronic viral hepatitis and cirrhotic groups.

Median serum galectin-3 levels were $319 \mathrm{pg} / \mathrm{ml}$ (242-375) among HCC patients, $348.5 \mathrm{pg} / \mathrm{ml}$ (236.5-445) among cirrhotic patients, $174.1 \mathrm{pg} / \mathrm{ml}$ (143-211) among chronic hepatitis C patients and $142.5 \mathrm{pg} / \mathrm{ml}$ (116.4-187) among normal subjects. The serum galectin-3 level was significantly higher in HCC patients than in those with chronic hepatitis $C$ $(p<0.001)$ and in normal subjects $(p<0.001)$. Also it was significantly higher among cirrhotic patients than in patients with chronic hepatitis $\mathrm{C}(p<0.001)$ and healthy persons $(p<0.001)$. On comparing HCC and cirrhotic patients, serum galectin-3 levels were not significantly different $(p=0.926)$. There was no statistically significant difference in galectin-3 levels between chronic HCV patients and healthy persons $(p=0.29)$ (Fig. 1). Table 1 shows the comparison between the studied groups according to demographic data, basic laboratory tests and galectin-3 levels.

Within the cirrhotic group, only one patient was in Child-Pugh class A while 31 patients were in ChildPugh class $\mathrm{B}$ and 8 patients were in Child-Pugh class $\mathrm{C}$, while the MELD score ranged from 7 to 35 (median $=15)$. The galectin-3 level was $355 \mathrm{pg} / \mathrm{ml}$ in the single Child-Pugh class A patient while the median galectin-3 level was $338 \mathrm{pg} / \mathrm{ml}$ among ChildPugh class B patients and $352.5 \mathrm{pg} / \mathrm{ml}$ among ChildPugh class C patients $(p=0.695)$.

\section{Discussion}

Hepatocellular carcinoma is usually asymptomatic [5]. Most HCC patients present with non-operable disease, and so early diagnosis is important to achieve a better prognosis $[3,5]$.

Many tumor markers have been suggested for HCC diagnosis, but few deserved to be used in clinical practice $[15,16]$.

Serum AFP is the traditional tumor marker used for HCC diagnosis $[5,17]$. However, serum AFP levels are normal in up to $30 \%$ of HCC patients, particularly in the early stage of the disease [18]. Recent studies showed no value for implementing AFP level follow-up in screening for HCC among cirrhotic patients [5]. On the basis of these considerations, new biomarkers with better performance are needed.

Galectin-3 is a member of the galectin family that is mainly produced by macrophages and is involved in various biological events including inflammation and angiogenesis [19]. Galectin-3 expression was found to be induced in HCC cases [20], which inhibits the immune response against tumor cells through stimulation of apoptosis of lymphocytes [21]. It is also reported that circulating galectin-3 could enhance cell migration and extracellular matrix invasion, facilitating tumor growth and spread [22, 23]. Moreover, galectin-3 increases the expression of vascular endothelial-cadherin, inducing neoangiogenesis [24].

In our study, on comparing HCC and cirrhotic patients serum galectin-3 levels were not significantly different $(p=0.926)$. This was different from what was found by Matsuda et al., who reported significantly higher levels of serum galectin-3 in HCC patients compared to cirrhotic patients $(p=0.03)$ [11].

In agreement with our study, a study which included HCC, cirrhotic and chronic viral hepatitis groups did not reveal a statistically significant difference in galectin-3 levels between HCC and cirrhotic patients ( $p=0.5$ ) but galectin-3 levels were significantly lower in the chronic viral hepatitis group as compared with cirrhosis and HCC groups $(p<0.001)$ [25]. Another study reported no significant difference between patients with HCC and patients with cirrhosis [26].

The sensitivity and specificity of galectin- 3 to predict $\mathrm{HCC}$ among patients with chronic viral hepatitis $\mathrm{B}$ were $80 \%$ and $93 \%$ respectively [27]. The present study found that galectin-3 levels were significantly higher in the HCC group than the chronic hepatitis $\mathrm{C}$ group without advanced fibrosis, but this cannot be helpful in clinical practice as chronic HCV patients almost always develop HCC on top of cirrhotic liver [28].

On the other hand, our results showed a significant difference in galectin-3 levels between cirrhotic patients and those with chronic hepatitis $\mathrm{C}$ without advanced fibrosis. As we did not perform liver biopsy in our study and our aim was to assess the possible role of 
Table 1. Comparison between studied groups according to demographic data, basic laboratory tests and galectin-3 levels

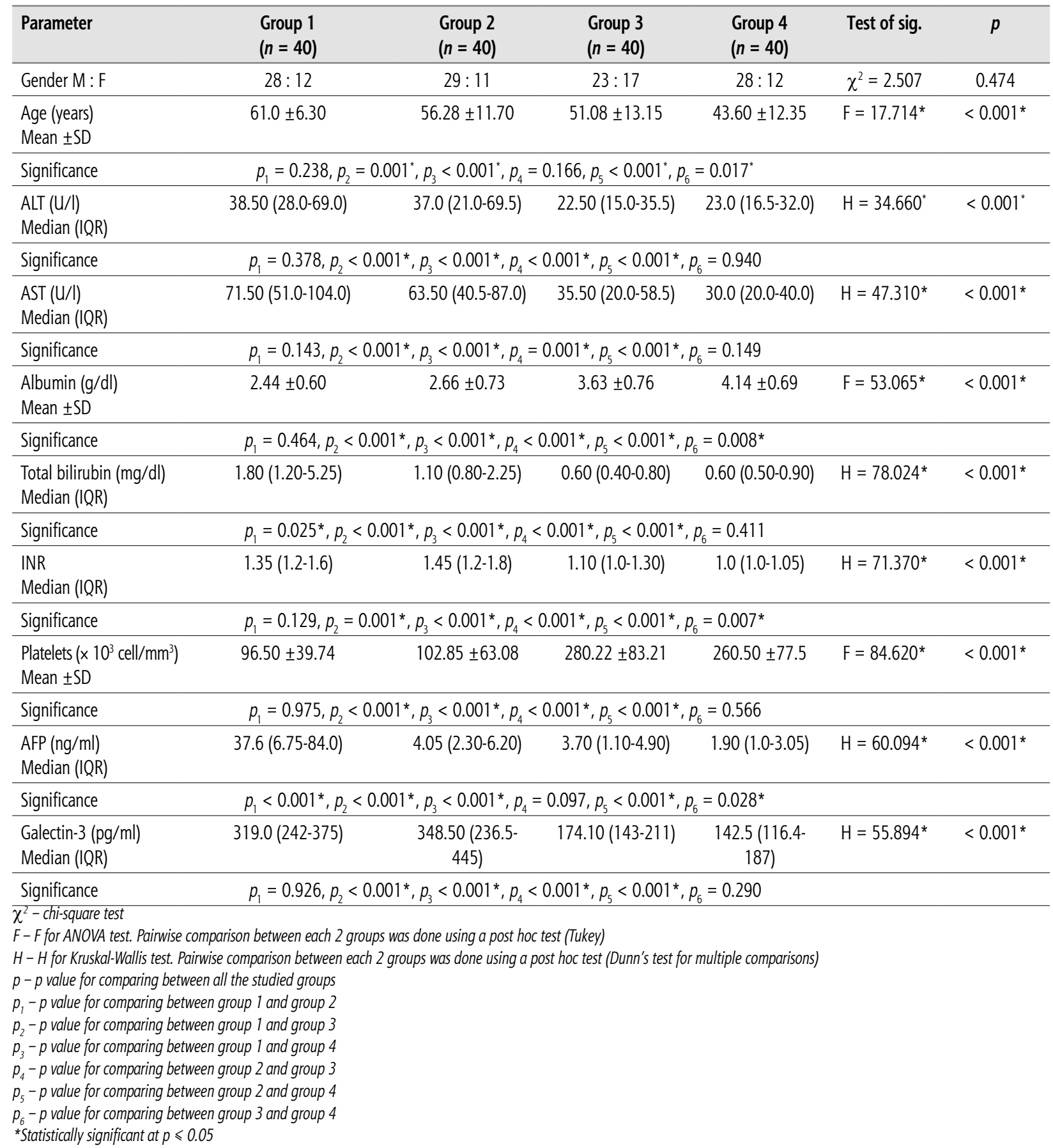

galectin-3 as a tumor marker among cirrhotic patients, we included in the cirrhosis group those with clinically evident cirrhosis, making it inappropriate to assess the role of galectin-3 levels in differentiating between various liver fibrosis stages. So we recommend further studies to explore this possible role.

The galectin-3 level was $355 \mathrm{pg} / \mathrm{ml}$ in the sole ChildPugh class A patient, while the median galectin-3 level among Child-Pugh class B patients was $338 \mathrm{pg} / \mathrm{ml}$ and $352.5 \mathrm{pg} / \mathrm{ml}$ among Child-Pugh class C patients. But as various Child-Pugh classes were not efficiently represented, we recommend researchers to further study levels of galectin-3 among different cirrhosis classes in representative samples.

\section{Conclusions}

Although the galectin-3 level is significantly higher among HCC patients than those with chronic hepatitis, it cannot be used as an additional method for sur- 
veillance of HCC among cirrhotic patients. Galectin-3 levels are significantly higher among patients with clinically evident cirrhosis than those with chronic hepatitis $\mathrm{C}$ without advanced fibrosis. We recommend researchers to study the role of galectin-3 in differentiating various stages of liver fibrosis.

\section{Acknowledgements}

The authors would like to acknowledge Dr. Ramy Ghazy, Assistant Lecturer at the High Institute of Public Health, Alexandria University, for his assistance in statistical analysis of the data.

\section{Disclosure}

The authors declare no conflict of interest.

\section{References}

1. Sagnelli E, Macera M, Russo A, et al. Epidemiological and etiological variations in hepatocellular carcinoma. Infection 2020; 48: 7-17.

2. Ibrahim AS, Khaled HM, Mikhail NN, et al. Cancer incidence in Egypt: results of the national population-based cancer registry program. J Cancer Epidemiol 2014; 2014: 437971.

3. Giannitrapani L, Zerbo M, Amodeo S, et al. The changing epidemiology of hepatocellular carcinoma: experience of a single center. Biomed Res Int 2020; 2020: 5309307.

4. Crissien AM, Frenette C. Current management of hepatocellular carcinoma. Gastroenterol Hepatol 2014; 10: 153-161.

5. Harris PS, Hansen RM, Gray ME, et al. Hepatocellular carcinoma surveillance: An evidence-based approach. World J Gastroenterol 2019; 25: 1550-1559.

6. Chou FC, Chen HY, Kuo CC, et al. Role of galectins in tumors and in clinical immunotherapy. Int J Mol Sci 2018; 19: 430.

7. Jiang SS, Weng DS, Wang QJ, et al. Galectin-3 is associated with a poor prognosis in primary hepatocellular carcinoma. J Transl Med 2014; 12: 273.

8. Shao Q, He J, Chen Z, Wu C. Prognostic role of galectins expression in patients with hepatic cancer: a meta-analysis. Medicine 2020; 99: e19622.

9. Wang M, Tian F, Ying W, Qian X. Quantitative proteomics reveal the anti-tumour mechanism of the carbohydrate recognition domain of Galectin-3 in Hepatocellular carcinoma. Sci Rep 2017; 7: 5189.

10. Zheng D, Hu Z, He F, et al. Downregulation of galectin- 3 causes a decrease in UPAR levels and inhibits the proliferation, migration and invasion of hepatocellular carcinoma cells. Oncol Rep 2014; 32: 411-418.

11. Matsuda Y, Yamagiwa Y, Fukushima K, et al. Expression of galectin-3 involved in prognosis of patients with hepatocellular carcinoma. Hepatol Res 2008; 38: 1098-1111.

12. Vallet-Pichard A, Mallet V, Nalpas B, et al. FIB-4: an inexpensive and accurate marker of fibrosis in HCV infection. comparison with liver biopsy and fibrotest. Hepatology 2007; 46: 32-36.

13. Peng Y, Qi X, Guo X. Child-Pugh versus MELD score for the assessment of prognosis in liver cirrhosis: a systematic review and meta-analysis of observational studies. Medicine 2016; 95: e2877.
14. An C, Rakhmonova G, Choi JY, et al. Liver imaging reporting and data system (LI-RADS) version 2014: understanding and application of the diagnostic algorithm. Clin Mol Hepatol 2016; 22: 296-307.

15. Ocker M. Biomarkers for hepatocellular carcinoma: What's new on the horizon? World J Gastroenterol 2018; 24: 3974-3979.

16. Lou J, Zhang L, Lv S, et al. Biomarkers for hepatocellular carcinoma. Biomark Cancer 2017; 9: 1-9.

17. Pascual S, Miralles C, Bernabé JM, et al. Surveillance and diagnosis of hepatocellular carcinoma: A systematic review. World J Clin Cases 2019; 7: 2269-2286.

18. Zhou L, Liu J, Luo F. Serum tumor markers for detection of hepatocellular carcinoma. World J Gastroenterol 2006; 12: 11751181.

19. Calvier L, Miana M, Reboul P, et al. Galectin-3 mediates aldosterone-induced vascular fibrosis. Arterioscler Thromb Vasc Biol 2013; 33: 67-75.

20. Fang QQ, Ni RZ, Xiao MB, et al. Serum and tissue expressions of galectin-3 in hepatocellular carcinoma and the clinical significances. Zhonghua Gan Zang Bing Za Zhi 2011; 19: 527-531.

21. Tummala KS, Brandt M, Teijeiro A, et al. Hepatocellular carcinomas originate predominantly from hepatocytes and benign lesions from hepatic progenitor cells. Cell Rep 2017; 19: 584600.

22. Fuchs BC, Hoshida Y, Fujii T, et al. Epidermal growth factor receptor inhibition attenuates liver fibrosis and development of hepatocellular carcinoma. Hepatology 2014; 59: 1577-1590.

23. Serizawa N, Tian J, Fukada H, et al. Galectin 3 regulates HCC cell invasion by RhoA and MLCK activation. Lab Invest 2015; 95: 1145-1156.

24. Guo XH, Liu LX, Zhang HY, et al. Insulin-like growth factor binding protein-related protein 1 contributes to hepatic fibrogenesis. J Dig Dis 2014; 15: 202-210.

25. Ulu M, Alacacioglu A, Yuksel E, et al. Prognostic significance of serum galectin-3 levels in patients with hepatocellular cancer and chronic viral hepatitis. Saudi J Gastroenterol 2015; 21: 47-50.

26. Eisa NH, Ebrahim MA, Ragab M, et al. Galectin-3 and matrix metalloproteinase-9: Perspective in management of hepatocellular carcinoma. J Oncol Pharm Pract 2015; 21: 323-330.

27. Liu T, Liu D, Liu R, et al. Discovering potential serological biomarker for chronic Hepatitis B Virus-related hepatocellular carcinoma in Chinese population by MAL-associated serum glycoproteomics analysis. Sci Rep 2017; 7: 38918.

28. Jindal A, Thadi A, Shailubhai K. Hepatocellular carcinoma: etiology and current and future drugs. J Clin Exp Hepatol 2019; 9: 221-232. 